\title{
Brain Activation Patterns Associated with the Effects of Fearful Distractors during Working Memory Maintenance in Patients with Schizophrenia
}

\author{
Jong-Il Park ${ }^{1,2}$, Gwang-Won Kim ${ }^{3}$, Gwang-Woo Jeong ${ }^{4}$, Jong-Chul Yang ${ }^{1,2}$ \\ ${ }^{1}$ Department of Psychiatry, Chonbuk National University Medical School, Jeonju, ${ }^{2}$ Research Institute of Clinical Medicine of Chonbuk National \\ University-Biomedical Research Institute of Chonbuk National University Hospital, Jeonju, ${ }^{3}$ Research Institute for Medical Imaging, Chonnam \\ National University Hospital, Gwangju, ${ }^{4}$ Department of Radiology, Chonnam National University Hospital, Chonnam Natioanl University \\ Medical School, Gwangju, Korea
}

\begin{abstract}
Objective: The neural correlates underlying the effects of emotional distraction during working memory (WM) tasks in patients with schizophrenia have yet to be clearly identified. Thus, the present study employed functional magnetic resonance imaging (fMRI) to investigate the effects of emotional distraction involving fear during WM maintenance in patients with schizophrenia.

Methods: This study included 17 patients with schizophrenia who were diagnosed based on Diagnostic and Statistical Manual of Mental Disorders fourth edition, text revision (DSM-IV-TR) criteria and 17 matched healthy controls. Event-related FMRI data were acquired while the participants performed a delayed-response WM task that included neutral and fearful distractors.

Results: Patients with schizophrenia may have tried to maintain WM function during the presentation of task-irrelevant fearful distractors that induced interruption and required attention. Compared to healthy controls, the schizophrenia patients exhibited significantly increased activity in the dorsolateral prefrontal cortex, medial prefrontal cortex, superior temporal gyrus, middle temporal gyrus, insula, hippocampus, caudate nucleus, and postcentral gyrus in a delayed-response WM task when presented with fearful relative to neutral distractors. In addition to its series of increased brain activations, prefrontal areas exhibited interconnections with more caudal brain regions, including temporal areas and the hippocampus and insula.

Conclusion: The present study identified specific brain areas associated with the interaction between emotional regulation and cognitive functioning during fearful distractors presented while patients with schizophrenia performed a WM maintenance task. These findings further the current understanding of the neural correlates underlying the effects of emotional distraction on cognitive functioning in patients with schizophrenia.
\end{abstract}

KEY WORDS: Emotional distractor; Functional magnetic resonance imaging; Schizophrenia; Working memory.

\section{INTRODUCTION}

Schizophrenia is a severe neuropsychiatric disease characterized by disruptions in cognitive and emotional functioning. During the course of schizophrenia, patients exhibit cognitive deficits that affect the speed of process-

Received: May 11, 2017 / Revised: July 19, 2017

Accepted: July 20, 2017

Address for correspondence: Jong-Chul Yang, MD, PhD Department of Psychiatry, Chonbuk National University Medical School, 20 Geonji-ro, Deokjin-gu, Jeonju 54907, Korea

Tel: +82-63-250-2580, Fax: +82-63-275-3157

E-mail: yangjc@jbnu.ac.kr

ORCID: https://orcid.org/0000-0002-7701-2718 ing, attention/vigilance, working memory (WM), verbal learning and memory, visual learning and memory, reasoning, problem solving, and verbal comprehension. ${ }^{1)}$ Cognitive deficits are associated with poor functional outcome in schizophrenia ${ }^{2)}$ and other psychiatric disorders. ${ }^{3)}$ WM tasks require the maintenance and manipulation of information relevant to current tasks over short periods of time while engaging in goal-directed behavior in the face of interfering processes and distractions. ${ }^{4)}$ This type of cognitive control involves two primary processes: 1 ) operations that allow for the active maintenance of goals and goal-relevant information in the mind (WM) and 2) operations that keep goal-irrelevant information out of the

(ㄷ) This is an Open-Access article distributed under the terms of the Creative Commons Attribution Non-Commercial License (http://creativecommons.org/licenses/by-nc/4.0) which permits unrestricted non-commercial use, distribution, and reproduction in any medium, provided the original work is properly cited. 
mind (cognitive inhibition). ${ }^{5)}$ In particular, emotional stimuli distract from and challenge the ability to maintain focus on goal-relevant information ${ }^{6)}$ and, subsequently, can impair cognitive performance.

Schizophrenia is often characterized by the abnormal filtering of thoughts and perceptions that causes deficits in the ability to determine which stimulus is real. Oltmanns and Neale ${ }^{7)}$ found that patients with schizophrenia show reduced digit-span performance under conditions of distraction, and it appears that the cognitive impairments associated with schizophrenia are related to interference deficits that play an important role in optimal WM functioning. ${ }^{8)}$ Moreover, these issues might be associated with deficits in maintaining focus on goal-relevant information during the presentation of confusing distractors. Inhibitory control over task-irrelevant information is thought to play a crucial role in cognitive control and has also been associated with the impairments in cognitive functioning observed in schizophrenia. ${ }^{9,10)}$ However, the effects of distraction due to interference from fear-related emotional stimuli during the delay interval of a WM task have received much less attention. Thus, it might be important to further elucidate the higher cognitive control functions associated with schizophrenia.

The present functional neuroimaging study conducted event-related functional magnetic resonance imaging (fMRI) assessments while participants performed a delayed-response WM task that included neutral and fearful distractors. Delayed-response WM tasks have been used to investigate the neural correlates of WM maintenance during the presentation of task-irrelevant distractors in the interval between encoding and retrieval. ${ }^{11)}$ Although fMRI studies using delayed-response WM tasks have been conducted in healthy controls, ${ }^{5,11)}$ few studies have assessed the neural mechanisms underlying the effects of fear-related emotional distraction on the cognitive function of patients with schizophrenia during such tasks. To the best of our knowledge, the present fMRI study is the first to examine the regional brain differences that are directly associated with the effects of fear-related emotional distraction during a delayed WM task in patients with schizophrenia. The present findings may provide novel evidence demonstrating differences between healthy controls and patients with schizophrenia in terms of the neural correlates supporting inhibitory processes during a delayed WM task.

\section{METHODS}

\section{Subjects}

The present study included 17 patients with schizophrenia (mean age, 30.9 \pm 8.7 years) and 17 healthy controls (mean age, $32.8 \pm 8.6$ years) who underwent an fMRI procedure in a 3.0 Tesla Magneton Verio MR Scanner (Siemens Medical Solutions, Erlangen, Germany); all subjects were right-handed (Table 1). All patients were diagnosed based on Diagnostic and Statistical Manual of Mental Disorders fourth edition, text revision (DSM-IVTR) criteria according to the Structured Clinical Interview for Axis I DSM-IV Disorders (SCID-I). ${ }^{12)}$ None of the patients had any other psychiatric disorders, and none of the healthy control subjects had a history of neurological or psychiatric illness. The severity of the symptoms of the patients was evaluated with the Positive and Negative Syndrome Scale (PANSS) ${ }^{13,14)}$ and the Clinical Global Impression (CGI) ${ }^{15)}$ scale (Table 1). All schizophrenia patients had prescription with atypical antipsychotics, and 11 had coprescription with other psychotropic medications (Table 2). The subjects practiced the task paradigm prior to MR scanning and were instructed to sustain attention during the task. All subjects provided written informed consent, and the study protocol was approved by Institutional Review Board of Chonbuk National University Hospital (No. CBIRB0907-65).

Table 1. Characteristics of patients with schizophrenia and healthy controls

\begin{tabular}{lccc}
\hline \multicolumn{1}{c}{ Characteristic } & $\begin{array}{c}\text { Patient with } \\
\text { schizophrenia } \\
(\mathrm{n}=17)\end{array}$ & $\begin{array}{c}\text { Healthy } \\
\text { control } \\
(\mathrm{n}=17)\end{array}$ & $p$ value \\
\hline Age (yr) & $31.1 \pm 9.0$ & $32.8 \pm 8.6$ & $0.512^{*}$ \\
Sex (male/female) & $9 / 8$ & $9 / 8$ & $1.000^{\dagger}$ \\
Handedness (\% right) & 100 & 100 & $1.000^{\dagger}$ \\
Education (yr) & $14.2 \pm 2.6$ & $14.6 \pm 2.1$ & $0.814^{*}$ \\
Duration of illness (yr) & $8.5 \pm 5.9$ & - & - \\
CGl & $3.8 \pm 1.0$ & - & - \\
PANSS & & - & - \\
Total score & $72.7 \pm 18.0$ & - & - \\
Positive symptoms & $16.5 \pm 5.7$ & - & - \\
Negative symptoms & $19.5 \pm 6.6$ & - & - \\
General psychopathology & $36.7 \pm 6.8$ & - & - \\
\hline
\end{tabular}

Values are presented as mean \pm standard deviation, number only, or $\%$ right.

CGI, Clinical Global Impression; PANSS, Positive and Negative Syndrome Scale.

*Independent-samples $t$ test (Mann-Whitney $U$ test), ${ }^{\dagger}$ chi-square test. 


\section{Task Paradigms}

All subjects underwent $\mathrm{fMRI}$ scans while performing a delayed-response WM task (face recognition task) with fearful distractors. The WM task paradigm consisted of the following sequence of trials: encoding for 6 seconds, WM maintenance for 4 seconds, distractor for 6 seconds, button preparation for 2 seconds, retrieval for 2 seconds, and inter-trial interval for 12 seconds (Fig. 1). The human faces (half male, half female) were selected from a high school yearbook, converted to black-and-white, and cropped to an oval shape showing only the eyes, nose, mouth, and eyebrows. Emotional distractors (neutral and fearful pictures) were viewed to induce emotional responses in the participants. In total, 50 emotional distractor images were collected from the International Affective Picture System $(\text { IAPS })^{16)}$ and a variety of Internet websites. The neutral

Table 2. Psychotropic medications used by patients with schizophrenia

\begin{tabular}{cc}
\hline Psychotropic medication & Range of daily dosage $(\mathrm{mg})$ \\
\hline Antipsychotics & \\
Amisulpride $(n=5)$ & $400-1,600$ \\
Aripiprazole $(n=1)$ & 10 \\
Clozapine $(n=1)$ & 300 \\
Olanzapine $(n=2)$ & $5-15$ \\
Paliperidone $(n=5)$ & $6-15$ \\
Quetiapine $(n=2)$ & $50-100$ \\
Risperidone $(n=6)$ & $4-12$ \\
Other medications & \\
Benztropine $(n=3)$ & $1-2$ \\
Diazepam $(n=1)$ & 5 \\
Fluoxetine $(n=3)$ & $40-80$ \\
Lorazepam $(n=2)$ & $0.5-2.0$ \\
Trazodone $(n=2)$ & 50 \\
Valproic acid $(n=1)$ & 1,200 \\
\hline
\end{tabular}

pictures (e.g., scenic pictures) were designed to induce a comfortable feeling while the fearful pictures included photographs of life-threatening behaviors. A psychiatrist selected 20 neutral and 20 fearful pictures. All tasks in this $\mathrm{fMRI}$ paradigm were presented to the subjects using SuperLab software (Cedrus Co., San Pedro, CA, USA).

\section{fMRI Data Acquisition}

All fMRI data were acquired using a 3.0 Tesla Magneton Verio MR Scanner with a bird-cage head coil. A total of 25 axial slices parallel to the anterior commissure to posterior commissure (AC-PC) line were obtained using a gradient echo planar pulse sequence with the following parameters: repetition time (TR)/echo time $(T E)=2,000 \mathrm{~ms} / 30 \mathrm{~ms}$, flip angle $=90^{\circ}$, field of view $(\mathrm{FOV})=22 \times 22 \mathrm{~cm}$, matrix size $=64 \times 64$, and slice thickness $=5 \mathrm{~mm}$. Additionally, two phases of dummy scans were performed to circumvent unstable fMRI signals. The high-resolution T1-weighted images ( $T R / T E=1,900 / 2.35$ $\mathrm{ms}$ ) had the following parameters: $\mathrm{FOV}=22 \times 22 \mathrm{~cm}$, matrix size $=256 \times 256$, and slice thickness $=5 \mathrm{~mm}$.

\section{Data Preprocessing and Analysis}

The fMRI data were analyzed with Statistical Parametric Mapping (SPM8; Wellcome Department of Cognitive Neurology, University College London, London, UK). Prior to statistical analysis, a slice-timing correction was performed on the fMRI data in which the images were realigned to the reference volume, spatially normalized to the standard echoplanar imaging template in the Montreal Neurological Institute space, and resampled to a resolution of $2 \times 2 \times 2 \mathrm{~mm}$. Finally, the images were

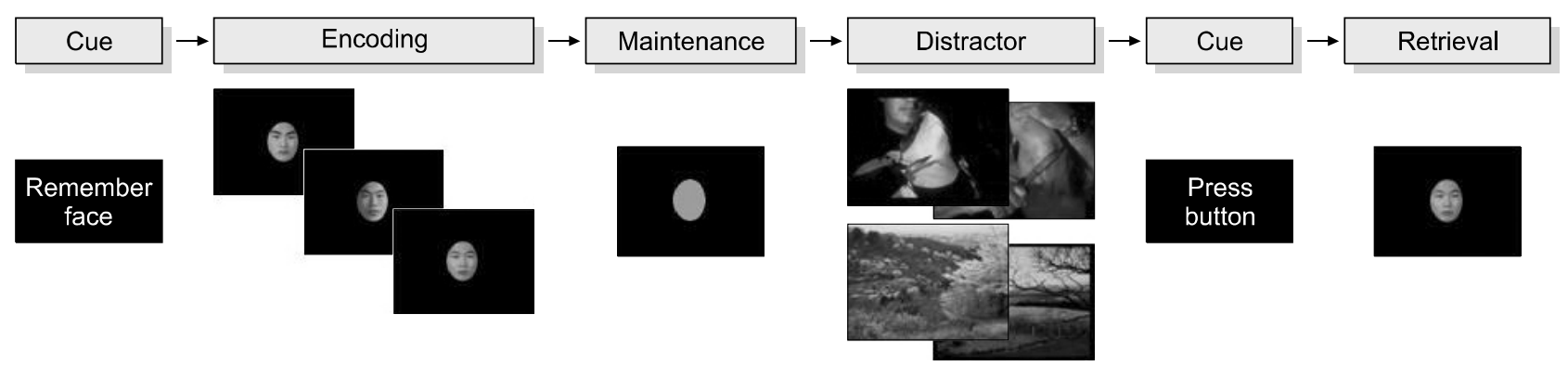

Fig. 1. Diagrams of the delayed-response working memory tasks with fearful and neutral distractors. In the encoding task, three different human faces appeared once. The subjects were instructed to encode and maintain a working memory (WM) of the presented human face and then look at either the neutral or the fearful distractor while maintaining the WM. During the retrieval period, either the face presented in the encoding task or a novel face was presented (50\% were presented with an encoding face and 50\% were presented with a novel face), and the response to the probe for the previously presented human face or the novel face was recorded. 
smoothed with an 8-mm full-width-half-maximum Gaussian filter.

The preprocessed data were analyzed using the standard general linear model approach within SPM8; the data from the distractor phase were used for the imaging analyses. To analyze individual blood-oxygen-level-dependent (BOLD) signals, independent $t$ tests were performed for the rest and activation conditions (neutral pictures and fearful pictures, respectively). For the within-group comparisons of the healthy controls and schizophrenia patients, differential brain activation maps that corresponded to the contrasts of fearful pictures versus neutral pictures were obtained using paired $t$ tests $(p<0.001)$. Two-sample $t$ tests (uncorrected, $p<0.005$ ) were conducted to compare the differential brain activation patterns between the healthy controls and the patients; activations that included more than 10 contiguous voxels for cluster size were reported. All data from incorrect trials of the facial recognition task were excluded from all statistical analyses.

\section{RESULTS}

\section{Demographic and Clinical Characteristics}

Table 1 shows the demographic and clinical characteristics of the schizophrenia patients and healthy controls, including duration of illness (8.5 \pm 5.9 years), score on the CGI scale (3.8 \pm 1.0$)$, and scores on the PANSS (total score, $72.7 \pm 18.0$; positive symptoms, $16.5 \pm 5.7$; negative symptoms, 19.5 \pm 6.6 ; and general psychopathology, 36.7 \pm 6.8 ). There were no significant differences in age, gender, handedness, or education between the two groups (Table 1).

On the face recognition task, the scores for the neutral distractors (10 trials) were not significantly different between in patients with schizophrenia and healthy controls (59.4 $\pm 14.3 \%$ and $66.5 \pm 14.1 \%, p=0.158)$, whereas the scores for the fearful distractors (10 trials) were significantly lower in patients with schizophrenia than in healthy controls $(56.5 \pm 12.7 \%$ and $68.8 \pm 11.1 \%, p=$ 0.005), respectively. The reaction times to both neutral distractors and fearful distractors were significantly slower in patients with schizophrenia than in healthy controls

Table 3. Brain regions in healthy controls and patients with schizophrenia showing increased activation in response to the fearful relative to the neutral distractor $(p<0.001)$

\begin{tabular}{|c|c|c|c|c|c|c|c|c|}
\hline \multirow{2}{*}{ Brain area } & \multicolumn{4}{|c|}{ Healthy control } & \multicolumn{4}{|c|}{ Patient with schizophrenia } \\
\hline & \multicolumn{3}{|c|}{ MNI coordinates $1(x, y, z)$} & $t$ value & \multicolumn{3}{|c|}{ MNI coordinates $(x, y, z)$} & $t$ value \\
\hline \multicolumn{9}{|l|}{ Frontal lobe } \\
\hline Medial PFC & 12 & 52 & 44 & 4.90 & 8 & 50 & 42 & 3.81 \\
\hline Dorsolateral PFC & 50 & 46 & 2 & 4.65 & - & - & - & - \\
\hline Ventrolateral PFC & 52 & 44 & -2 & 5.16 & -54 & 26 & 30 & 4.54 \\
\hline Orbitofrontal gyrus & - & - & - & - & 28 & 16 & -20 & 5.50 \\
\hline Precentral gyrus & 56 & 6 & 46 & 4.77 & 42 & 0 & 36 & 4.20 \\
\hline \multicolumn{9}{|l|}{ Temporal lobe } \\
\hline Superior temporal gyrus & - & - & - & - & -56 & -46 & 12 & 7.86 \\
\hline Middle temporal gyrus & -48 & -65 & -1 & 7.51 & 46 & -72 & -4 & 11.39 \\
\hline Inferior temporal gyrus & 44 & -52 & -16 & 10.08 & -42 & -74 & -2 & 12.33 \\
\hline Fusiform gyrus & 42 & -52 & -16 & 9.94 & -40 & -75 & -6 & 9.60 \\
\hline \multicolumn{9}{|l|}{ Parietal lobe } \\
\hline Superior parietal gyrus & 28 & -72 & 46 & 6.31 & - & - & - & - \\
\hline Angular gyrus & 29 & -68 & 46 & 5.21 & - & - & - & - \\
\hline Postcentral gyrus & - & - & - & - & -38 & -38 & 66 & 4.56 \\
\hline \multicolumn{9}{|l|}{ Occipital lobe } \\
\hline Superior occipital gyrus & 28 & -73 & 46 & 6.21 & - & - & - & - \\
\hline Middle occipital gyrus & 42 & -84 & -2 & 8.66 & -42 & -74 & -1 & 12.30 \\
\hline Inferior occipital gyrus & 42 & -82 & -3 & 8.55 & 48 & -74 & -6 & 11.63 \\
\hline \multicolumn{9}{|l|}{ Limbic lobe } \\
\hline Hippocampus & 26 & -8 & -18 & 5.67 & -18 & -30 & -4 & 4.50 \\
\hline Parahippocampal gyrus & 24 & -2 & -26 & 4.38 & 22 & 0 & -26 & 5.83 \\
\hline Amygdala & 26 & -2 & -25 & 4.24 & 24 & 2 & -26 & 5.16 \\
\hline Insula & - & - & - & - & 28 & 16 & -19 & 5.20 \\
\hline
\end{tabular}

MNI, Montreal Neurological Institute; PFC, prefrontal cortex. 
$(1,393.9 \pm 175.1 \mathrm{~ms}$ vs. $1,170.5 \pm 159.8 \mathrm{~ms}$ for neutral distractors, $p<0.001,1,545.7 \pm 226.4 \mathrm{~ms}$ vs. $1,260.9 \pm 186.6$ ms for fearful distractors, $p<0.001)$. The patients performed more poorly in response to both the neutral and fearful distractors relative to the healthy controls.

\section{Differential Activation Patterns between the Neutral and Fearful Distractors}

The healthy control group showed significantly stronger activity in response to the fearful distractors than to the neutral distractors in a variety of brain areas, including the medial prefrontal cortex (mPFC), dorsolateral PFC (DLPFC), ventrolateral PFC (VLPFC), middle temporal gyrus (MTG), inferior temporal gyrus (ITG), fusiform gyrus, superior parietal gyrus, angular gyrus, superior/middle/inferior occipital gyri (SOG, MOG, and IOG, respectively), hippocampus, parahippocampal gyrus, and amygdala during the delayed-response WM task ( $p<0.001$; Table 3, Fig. 2). Similar to the healthy controls, the schizophrenia patients showed significantly stronger activity in response to the fearful distractors than to the neutral distractors in several brain areas, including the mPFC, VLPFC, orbitofrontal cortex (OFC), superior temporal gyrus (STG), MTG, ITG, fusiform gyrus, MOG, IOG, hippocampus, parahippocampal gyrus, amygdala, and insula $(p<0.001$; Table 3, Fig. 2).
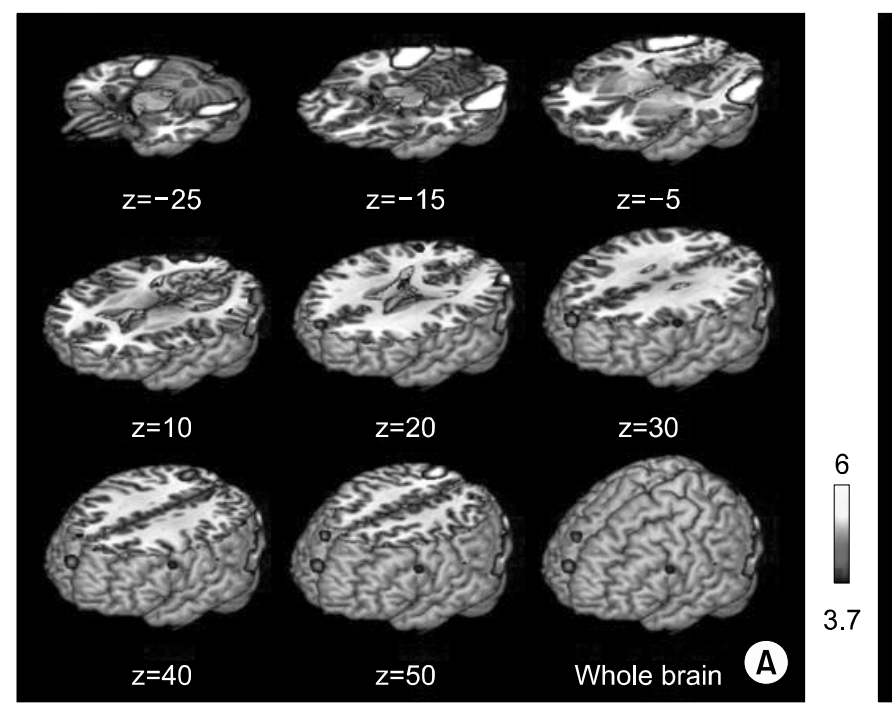

Fig. 2. Brain regions predominantly showing increased activation to the fearful relative to the neutral distractor $(p<0.001)$. (A) Healthy controls; (B) schizophrenia patients.

\section{Inter-group Differences in the Activation Patterns Associated with Fearful Distractors vs. Neutral Distractors}

Compared to the healthy controls, the patients with schizophrenia showed significant increases in activity in the DLPFC, mPFC, STG, MTG, insula, hippocampus, caudate nucleus, and postcentral gyrus $(p<0.005$; Table 4 , Fig. 3). These brain areas are potentially related to the maintenance of goal-relevant information and the neglect of goal-irrelevant information during interference related

Table 4. Brain regions showing increased activation in response to the fearful relative to the neutral distractor $(p<0.005)$

\begin{tabular}{lrrrr}
\hline \multicolumn{1}{c}{ Brain areas } & $t$ value & \multicolumn{2}{c}{ MNI coordinates $(x, y, z)$} \\
\hline Controls $>$ schizophrenia & & & & \\
Superior occipital gyrus & 4.84 & 30 & -70 & 44 \\
Superior parietal gyrus & 4.82 & 26 & -69 & 48 \\
Middle occipital gyrus & 4.49 & 32 & -72 & 38 \\
Precuneus & 3.76 & 24 & -60 & 30 \\
Fusiform gyrus & 3.74 & -34 & -40 & -22 \\
Precentral gyrus & 3.10 & -56 & 12 & 34 \\
Schizophrenia $>$ controls & & & & \\
Superior temporal gyrus & 4.66 & -58 & -44 & 16 \\
Postcentral gyrus & 4.02 & -31 & -34 & 58 \\
Insula & 3.81 & -26 & 26 & 12 \\
Middle temporal gyrus & 3.48 & 60 & -64 & 8 \\
Hippocampus & 3.17 & -32 & -32 & -4 \\
Medial PFC & 3.16 & 11 & 38 & 38 \\
Dorsolateral PFC & 3.11 & -32 & 14 & 60 \\
Head of caudate nucleus & 2.95 & -16 & 12 & 16 \\
\hline
\end{tabular}

MNI, Montreal Neurological Institute; PFC, prefrontal cortex.

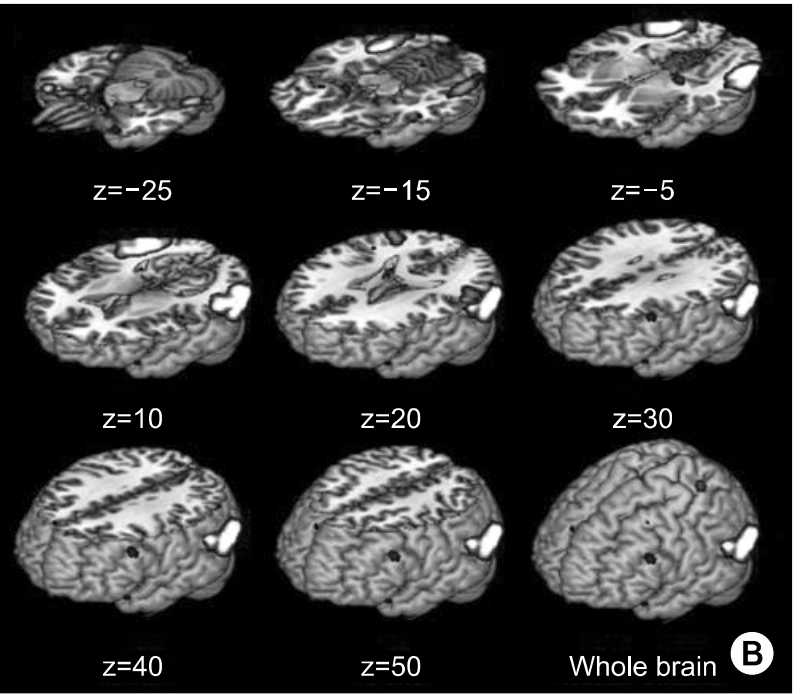



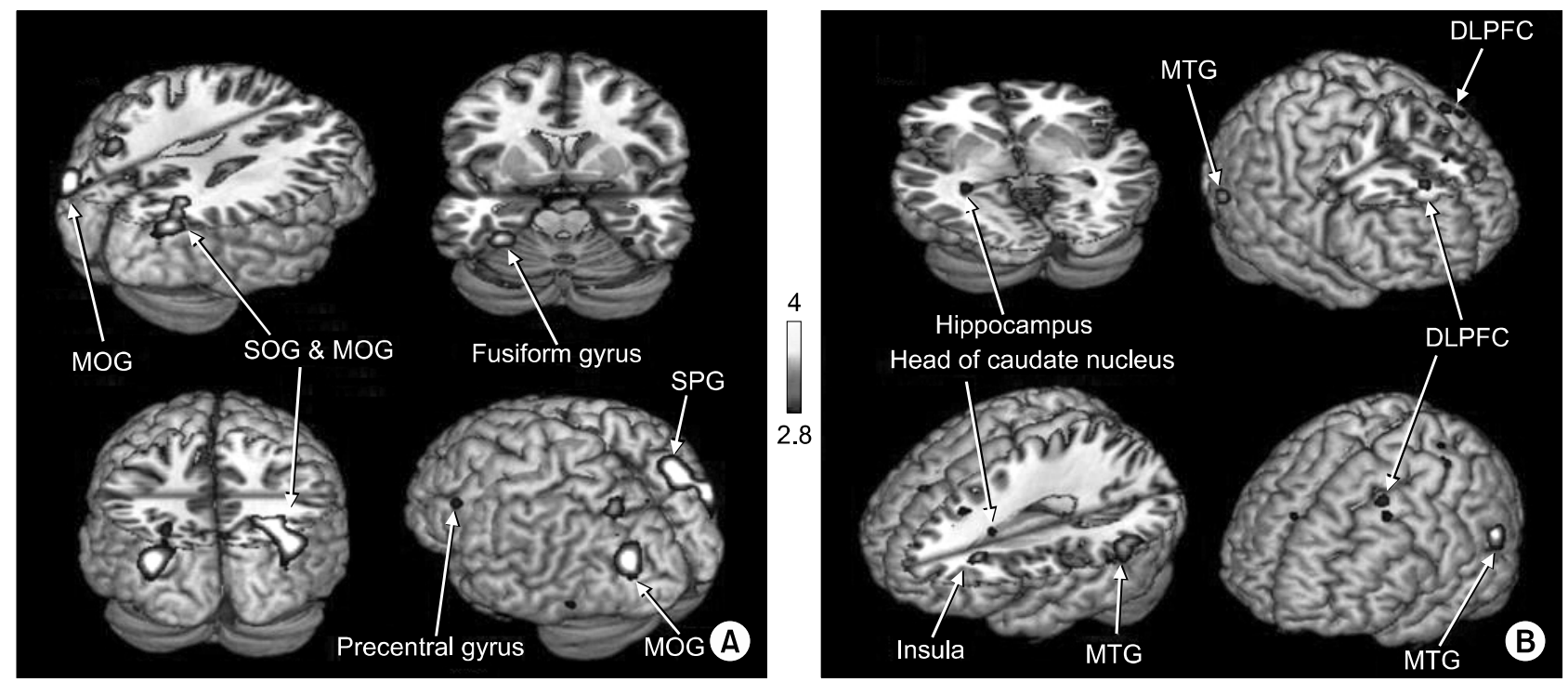

Fig. 3. Brain regions predominantly showing increased activation to the fearful relative to the neutral distractor $(p<0.005)$. (A) Healthy controls $>$ schizophrenia patients; (B) schizophrenia patients > healthy controls.

MOG, middle occipital gyri; SOG, superior occipital gyri; SPG, superior parietal temporal gyrus; MTG, middle temporal gyrus; DLPFC, dorsolateral prefrontal cortex.

to fearful stimuli. Compared to the patients with schizophrenia, the healthy controls showed significant increases in activity in the SOG, superior parietal gyrus, MOG, precuneus, fusiform gyrus, and precentral gyrus $(p<0.005$; Table 4, Fig. 3).

\section{DISCUSSION}

The present study aimed to determine the neural correlates associated with the effects of fearful emotional distractors during WM maintenance during a delayed-response task performed by patients with schizophrenia. During the face recognition task, both healthy controls and patients with schizophrenia attempted to maintain focus on goal-relevant information in the presence of interference due to emotional distraction. ${ }^{11)}$ The scores of patients with schizophrenia on the face recognition task with the fearful distractors were significantly lower than those of the healthy controls, which the patients with schizophrenia showed impairment in WM maintenance for fearful distractors. Accordingly, the present study identified brain regions relevant to WM maintenance, emotion, and cognitive inhibition that show increased activation during performance of a task (Table 3). In particular, compared to healthy controls, patients with schizophrenia showed significantly increased activity in the
DLPFC, mPFC, STG, MTG, insula, and hippocampus during the delayed-response WM task when presented with fearful pictures relative to neutral pictures (Table 4). The WM dysfunction in schizophrenia might be directly linked to deficits in the ability to maintain focus on goal-relevant information $^{17)}$ in the presence of fearful distractors.

Compared to healthy controls, patients with schizophrenia showed increases in activity in the DLPFC and mPFC during the delayed-response WM task. According to neuroimaging research, frontal lobe dysfunction is a cardinal feature of schizophrenia, and it is widely known that the DLPFC plays a major role in WM tasks. ${ }^{18)}$ Although the DLPFC was more activated in the patients with schizophrenia than in the healthy controls, the patients performed the WM task with less accuracy and had longer reaction times than healthy controls. Thus, increased DLPFC activation does not always correspond to enhanced WM performance and may reflect the inefficiency of this region in patients with schizophrenia. Accordingly, although the patients performed less accurately than the healthy controls during fearful emotional interference, the DFLPC of the patients may have had to work harder to respond to the task demands due to their WM capacity. Weinberger et al. ${ }^{19)}$ proposed that, even when patients with schizophrenia are able to keep up 
with WM demands during the Wisconsin Card Sort Test, they perform less efficiently than normal controls. Thus, the present findings are consistent with a series of previous studies. Several neuroimaging studies have suggested that DLPFC activation during WM tasks in patients with schizophrenia is due to the inefficiency of the DLPFC. ${ }^{20,21)}$ Potkin et al. ${ }^{17)}$ found that subjects with schizophrenia perform significantly worse, with less accuracy and longer reaction times, during the Sternberg Item Recognition Paradigm and that schizophrenic subjects exhibit significantly greater activation in the DLPFC than healthy controls.

In addition to the activation of the DLPFC, the MPFC showed increased activation in patients with schizophrenia compared to healthy controls in the present study. It is possible that the increased activations in the DLPFC and mPFC in the patients with schizophrenia were the result of affective-cognitive interactions during WM maintenance when presented with emotional distractors. This population may experience dysfunction in the regulation and filtering of emotional distractors. Indeed, models of affective-cognitive interactions, which were first implemented in depressed patients, have shown increased emotional distractibility and an impaired ability to maintain focus on relevant information in clinical populations. ${ }^{22)}$ Affective - cognitive interactions are primarily constituted by interaction between the dorsal executive system and the ventral affective system. The dorsal executive system, including the DLPFC and lateral parietal cortex, is critical for the active maintenance of goalrelevant information processing during $\mathrm{WM}_{1}^{23,24)}$ whereas the ventral emotional system, including the VLPFC, mPFC, and amygdala, is critical for emotional processing. ${ }^{25-27)}$ Taken together, these findings suggest that the deficits in executive function observed in patients with schizophrenia related to fearful emotional distractors might be explained by a failure to suppress the affective division of the mPFC. For example, Whitfield-Gabrieli et $a /{ }^{28)}$ identified hyperactivity (reduced task-related suppression) in the MPFC and hyperconnectivity between the mPFC and regions of the default mode network during a WM task in patients with schizophrenia and their unaffected relatives; these changes were correlated with inferior WM performance in both groups. The present findings may indicate that WM dysfunction in patients with schizophrenia results from inefficiency within the DLPFC and the failure of the mPFC to suppress emotional information.

The present study also showed that, compared to healthy controls, patients with schizophrenia exhibited significant increases in activity in the STG and MTG during the delayed-response WM task in response to the fearful distractors relative to the neutral distractors. The STG plays an important role in WM performance in terms of integrating previous actions and successful outcomes into decision-making strategies. The increased activations in the STG and MTG in patients with schizophrenia may be the result of extra effort when trying to maintain focus on target faces while overcoming fearful emotional interference. The present results could provide further evidence supporting the role of temporal lobe dysfunction in schizophrenia. Frith et al. ${ }^{29)}$ found that patients with chronic schizophrenia failed to show expected deactivation in the superior temporal cortex during a verbal fluency task, and Walter et al. ${ }^{30)}$ suggested that patients with schizophrenia might adapt to WM dysfunction while fulfilling task-related requirements via compensatory activation of the STG. Schizophrenia patients exhibit increased temporal activation relative to both healthy controls and patients with major depression during WM tasks with a high cognitive load, which indicates that increased activation in the temporal cortex is specific to patients with schizophrenia. ${ }^{30)}$ Similarly, Crossley et al. ${ }^{31)}$ observed superior temporal lobe dysfunction and frontotemporal dysconnectivity in first-episode psychosis patients using Dynamic Causal Modeling and fMRI. These authors reported that the STG engages differently in healthy controls, prodromal schizophrenia patients, and first-episode schizophrenia patients such that there is a deactivation of the STG during the N-back task in healthy controls, whereas first-episode schizophrenia patients show activation in this area, and prodromal schizophrenia patients exhibit responses that are intermediate to those of the other two other groups. In addition to previous findings showing temporal dysfunction in schizophrenia, the our results suggest that increased activation in the STG of schizophrenia patients during interference involving emotional distraction may be due to failed deactivation in the STG.

The present findings also revealed that the patients with schizophrenia exhibited increased activity in the insula and hippocampus during the delayed-response WM task 
with fearful distractors relative to the healthy controls. Assuming that the subjects viewed the photographs of life-threatening behaviors during the WM task, the activation in these two areas could be related to unpleasant emotions, such as fear, anxiety, or disgust. Several subcortical structures, including the thalamus, basal ganglia, amygdala, and hippocampus, are thought to regulate the compensatory response in schizophrenic patients during WM tasks as these regions may recruit a more extensive network. ${ }^{21,32)}$ Several studies have suggested that the hippocampus is responsive to unpleasant emotions ${ }^{33,34)}$ and that the insula is activated during the assessment of emotionally aversive states, including fear and disgust. ${ }^{35)}$ Moreover, insular activation is associated with awareness of threats, ${ }^{36)}$ self-relatedness, ${ }^{37)}$ and interoceptive awareness. ${ }^{38)}$ The insula is bi-directionally connected with the $\mathrm{STG}^{39)}$ which is bilaterally activated during WM performance during the assessment and action selection stages of decision-making. ${ }^{40)}$

Based on the results discussed so far in this paper, task-irrelevant fearful distractors during WM maintenance interrupt task performance and demand attention since the subjects initialize and make decisions regarding the special significance of the distracting stimuli. These series of activities might not be limited to the prefrontal area but may also include the interconnected activations of various regions, such as the temporal area, hippocampus, and insula. The prefrontal area plays major roles in the maintenance of focus on target faces, inhibition of goal-irrelevant information, and solving of problems during WM tasks with distractors. In the present study, the superior temporal gyri in patients with schizophrenia failed to show the expected deactivation, and the hippocampus and insula were associated with the fearful emotional responses caused by the distractors. Thus, the dysfunction observed in the patients with schizophrenia during the WM task might be correlated with the abovementioned brain areas.

The present study has several limitations that should be noted. First, there were a limited number of trials in the WM task since head movements could not be controlled, as the experimental time extended during the fMRI scan. Second, accuracy on the face recognition task was evaluated according to responses made within 2 seconds in the retrieval phase, and this time period may have been too short for the patients with schizophrenia to accurately respond. Finally, the possibility of medication effects on the brain activation patterns associated with distraction during the delay interval of the WM task could not be excluded because the patients with schizophrenia had been taking psychotropic medications. Thus, whether the observed brain dysfunction in schizophrenia patients was primary or secondary to other cognitive dysfunctions or the influence of medication needs to be further elucidated.

To the best of our knowledge, the present study is the first to provide neuroimaging evidence of differences in the brain activation patterns of patients with schizophrenia and healthy controls that are associated with the effects of fearful distraction during the delay interval of a WM task. The present findings may further the current understanding of the neural mechanisms underlying the effects of emotional distraction on WM dysfunction in patients with schizophrenia.

\section{- Acknowledgments}

This paper was supported by Fund of Biomedical Research Institute, Chonbuk National University Hospital. The authors do not have any conflicts of interest concerning publication of the submitted manuscript.

\section{REFERENCES}

1. Nuechterlein KH, Barch DM, Gold JM, Goldberg TE, Green MF, Heaton RK. Identification of separable cognitive factors in schizophrenia. Schizophr Res 2004;72:29-39.

2. Tripathi A, Kar SK, Shukla R. Cognitive deficits in schizophrenia: Understanding the biological correlates and remediation strategies. Clin Psychopharmacol Neurosci 28018;16:7-17.

3. Woo YS, Rosenblat JD, Kakar R, Bahk WM, McIntyre RS. Cognitive deficits as a mediator of poor occupational function in remitted major depressive disorder patients. Clin Psychopharmacol Neurosci 2016;14:1-16.

4. Baddeley AD. Working memory. Oxford:Oxford University Press; 1986.

5. Dolcos F, Miller B, Kragel P, Jha A, McCarthy G. Regional brain differences in the effect of distraction during the delay interval of a working memory task. Brain Res 2007;1152: 171-181.

6. Ellis HC, Ashbrook PW. Resource allocation model of the effects of depressed mood states on memory. In: Fiedler $K$, Forgas JP, editors. Affect, cognition, and social behavior: new evidence and integrative attempts. Toronto:Hogrefe; 1988. p.25-43.

7. Oltmanns TF, Neale JM. Schizophrenic performance when 
distractors are present: attentional deficit or differential task difficulty? J Abnorm Psychol 1975;84:205-209.

8. Hahn B, Robinson BM, Kaiser ST, Harvey AN, Beck VM, Leonard CJ, et al. Failure of schizophrenia patients to overcome salient distractors during working memory encoding. Biol Psychiatry 2010;68:603-609.

9. Choi JW, Jeong BS, Kim JW. Dysfunction of the left dorsolateral prefrontal cortex is primarily responsible for impaired attentional processing in schizophrenia. Psychiatry Investig 2008;5:52-59.

10. Weiss EM, Golaszewski S, Mottaghy FM, Hofer A, Hausmann A, Kemmler G, et al. Brain activation patterns during a selective attention test-a functional MRI study in healthy volunteers and patients with schizophrenia. Psychiatry Res 2003;123:1-15.

11. Dolcos F, Diaz-Granados P, Wang L, McCarthy G. Opposing influences of emotional and non-emotional distracters upon sustained prefrontal cortex activity during a delayed-response working memory task. Neuropsychologia 2008;46:326-335.

12. First MB, Spitzer RL, Gibbon M, Williams JBW. Structured clinical interview for DSM-IV-TR Axis / disorders: Patient edition. New York:Biometrics Research Department, Columbia University;2005.

13. Kay SR, Fiszbein A, Opler LA. The positive and negative syndrome scale (PANSS) for schizophrenia. Schizophr Bull 1987; 13:261-276.

14. Yi JS, Ahn YM, Shin HK, An SK, Joo YH, Kim SH, et al. Reliability and validity of the Korean version of the positive and negative syndrome scale. J Korean Neuropsychiatr Assoc 2001:40:1090-1105.

15. Haro JM, Kamath SA, Ochoa S, Novick D, Rele K, Fargas A, et al. The Clinical Global Impression-Schizophrenia scale: a simple instrument to measure the diversity of symptoms present in schizophrenia. Acta Psychiatr Scand Supp/ 2003;(416): 16-23.

16. Lang PJ, Bradley MM, Cuthbert BN. International affective picture system (IAPS): Affective ratings of pictures and instruction manual. Gainesville, FL:NIMH, Center for the Study of Emotion \& Attention;2005.

17. Potkin SG, Turner JA, Brown GG, McCarthy G, Greve DN, Glover $\mathrm{GH}$, et al. Working memory and DLPFC inefficiency in schizophrenia: the FBIRN study. Schizophr Bull 2009; 35:19-31.

18. Barbey AK, Koenigs M, Grafman J. Dorsolateral prefrontal contributions to human working memory. Cortex 2013;49: 1195-1205.

19. Weinberger DR, Berman KF, Zec RF. Physiologic dysfunction of dorsolateral prefrontal cortex in schizophrenia. I. Regional cerebral blood flow evidence. Arch Gen Psychiatry 1986; 43:114-124.

20. Manoach DS, Press DZ, Thangaraj V, Searl MM, Goff DC, Halpern E, et al. Schizophrenic subjects activate dorsolateral prefrontal cortex during a working memory task, as measured by fMRI. Biol Psychiatry 1999;45:1128-1137.

21. Manoach DS, Gollub RL, Benson ES, Searl MM, Goff DC, Halpern E, et al. Schizophrenic subjects show aberrant fMRI activation of dorsolateral prefrontal cortex and basal ganglia during working memory performance. Biol Psychiatry 2000; 48:99-109.

22. Watts FN, MacLeod AK, Morris L. Associations between phenomenal and objective aspects of concentration problems in depressed patients. Br J Psychol 1988;79:241-250.

23. Courtney SM, Ungerleider LG, Keil K, Haxby JV. Transient and sustained activity in a distributed neural system for human working memory. Nature 1997;386:608-611.

24. Smith EE, Jonides J. Storage and executive processes in the frontal lobes. Science 1999;283:1657-1661.

25. Davidson RJ, Irwin W. The functional neuroanatomy of emotion and affective style. Trends Cogn Sci 1999;3:11-21.

26. Phan KL, Wager T, Taylor SF, Liberzon I. Functional neuroanatomy of emotion: a meta-analysis of emotion activation studies in PET and FMRI. Neuroimage 2002;16:331-348.

27. Zald DH. The human amygdala and the emotional evaluation of sensory stimuli. Brain Res Rev 2003;41:88-123.

28. Whitfield-Gabrieli S, Thermenos HW, Milanovic S, Tsuang MT, Faraone SV, McCarley RW, et al. Hyperactivity and hyperconnectivity of the default network in schizophrenia and in first-degree relatives of persons with schizophrenia. Proc Natl Acad Sci U S A 2009;106:1279-1284.

29. Frith CD, Friston KJ, Herold S, Silbersweig D, Fletcher P, Cahill $\mathrm{C}$, et al. Regional brain activity in chronic schizophrenic patients during the performance of a verbal fluency task. $\mathrm{Br} J$ Psychiatry 1995; 167:343-349.

30. Walter H, Vasic N, Höse A, Spitzer M, Wolf RC. Working memory dysfunction in schizophrenia compared to healthy controls and patients with depression: evidence from event-related fMRI. Neuroimage 2007;35:1551-1561.

31. Crossley NA, Mechelli A, Fusar-Poli P, Broome MR, Matthiasson P, Johns LC, et al. Superior temporal lobe dysfunction and frontotemporal dysconnectivity in subjects at risk of psychosis and in first-episode psychosis. Hum Brain Mapp 2009;30:4129-4137.

32. Tura E, Turner JA, Fallon JH, Kennedy JL, Potkin SG. Multivariate analyses suggest genetic impacts on neurocircuitry in schizophrenia. Neuroreport 2008;19:603-607.

33. Critchley $H$, Daly E, Phillips $M$, Brammer M, Bullmore E, Williams S, et al. Explicit and implicit neural mechanisms for processing of social information from facial expressions: a functional magnetic resonance imaging study. Hum Brain Mapp 2000;9:93-105.

34. Lange K, Williams LM, Young AW, Bullmore ET, Brammer MJ, Williams SC, et al. Task instructions modulate neural responses to fearful facial expressions. Biol Psychiatry 2003;53. 226-232.

35. Phillips ML, Young AW, Scott SK, Calder AJ, Andrew C, Giampietro $\vee$, et al. Neural responses to facial and vocal ex- 
pressions of fear and disgust. Proc Biol Sci 1998;265:18091817.

36. Critchley HD, Mathias CJ, Dolan RJ. Fear conditioning in humans: the influence of awareness and autonomic arousal on functional neuroanatomy. Neuron 2002;33:653-663.

37. Phan KL, Taylor SF, Welsh RC, Ho SH, Britton JC, Liberzon I. Neural correlates of individual ratings of emotional salience: a trial-related fMRI study. Neuroimage 2004;21:768-780.

38. Critchley HD, Wiens S, Rotshtein P, Ohman A, Dolan RJ.
Neural systems supporting interoceptive awareness. Nat Neurosci 2004;7:189-195.

39. Augustine JR. The insular lobe in primates including humans. Neurol Res 1985;7:2-10.

40. Paulus MP, Feinstein JS, Leland D, Simmons AN. Superior temporal gyrus and insula provide response and outcome-dependent information during assessment and action selection in a decision-making situation. Neuroimage 2005; 25:607-615. 